\title{
IMPACT OF ENVIRONMENTAL AND GENETIC FACTORS ON THE SCALE SHAPE OF ZEBRAFISH, DANIO RERIO (HAMILTON 1822): A GEOMETRIC MORPHOMETRIC STUDY
}

\author{
Á. Staszny,,${ }^{1,3}$ Enikö Havas, ${ }^{2}$ R. Kovács,,${ }^{1}$ B. Urbányi, ${ }^{1}$ \\ G. Paulovits, ${ }^{3}$ Dóra Bencsik, ${ }^{1}$ Á. Ferincz, ${ }^{4}$ T. Müller, ${ }^{1}$ A. Specziár, ${ }^{3}$ \\ KATALIN BAKOS ${ }^{1}$ and Zs. CSENKI ${ }^{1, *}$ \\ ${ }^{1}$ Department of Aquaculture, Faculty of Agricultural and Environmental Sciences, \\ Institute of Environmental \& Landscape Management, Szent István University, \\ Páter K. út 1, H-2100 Gödöllö, Hungary \\ ${ }^{2}$ Department of Zoology and Animal Ecology, Faculty of Agricultural and Environmental Sciences, \\ Szent István University, Páter K. út 1, H-2100 Gödöllö, Hungary \\ ${ }^{3}$ Centre for Ecological Research, Balaton Limnological Institute, Hungarian Academy of Sciences, \\ Klebelsberg Kuno út 3, H-8237 Tihany, Hungary \\ ${ }^{4}$ Department of Limnology, University of Pannonia, Egyetem u. 10, H-8201 Veszprém, Hungary
}

(Received: November 22, 2012; accepted: February 1, 2013)

Intraspecific morphological variability may reflect either genetic divergence among groups of individuals or response of individuals to environmental circumstances within the frame of phenotypic plasticity. Several studies were able to discriminate wild fish populations based on their scale shape. Here we examine whether the variations in the scale shape in fish populations could be related to genetic or environmental factors, or to both of them. In the first experiment, two inbred lines of zebrafish, Danio rerio (Hamilton 1822) reared under identical environmental conditions were compared. Secondly, to find out what effect environmental factors might have, offsprings were divided into two groups and reared on different diets for 12 weeks. Potential recovery of scales from an environmental effect was also assessed. Experimental groups could successfully be distinguished according to the shape of scales in both experiments, and the results showed that both genetic and environmental factors may notably influence scale shape. It was concluded that scale shape analysis might be used as an explanatory tool to detect potential variability of environmental influences impacting genetically homogeneous groups of fish. However, due to its sensitivity to environmental heterogeneity, the applicability of this technique in identifying intraspecific stock membership of fish could be limited.

Keywords: Landmark-based geometric morphometrics - phenotypic plasticity - shape analysis

\section{INTRODUCTION}

When phenotypes can clearly associate with specific genotypes then they may be used to separate genetically different populations or groups of individuals of a species. However, if environmental effects can be captured safely in the formation of a specific morphological character then this character may be used as a good and

*Corresponding author; e-mail address: Csenki.Zsolt@mkk.szie.hu 
simple indicator for distinguishing among individuals maintained under different environmental circumstances or, in general, monitor environmental impacts stressing the population under study.

In fishes, morphometric analysis is especially suitable to assess various genetic, environmental and physiological effects that hit the individuals [36]. Besides the genetic variability, effects of food availability [4, 16, 20] and type of food [5], temperature [2, 16, 31], or the presence of predators [3] on body shape have been reported. However, the process of taking a proper morphometric image of the whole body is highly stressful for fish, and therefore, the investigation of a structural component, variable enough to distinguish populations and easy to collect without permanently damaging the animal is more expedient [8]. Assuming a strong genetic definiteness, scales, similarly to other hard structural components like otolith [1,17] and in general bony structures [33], are regularly used to distinguish among species or even populations of fish [10-12, 22-24]. The examination of scales proved to be a practical and cheap tool to identify fish including archaeological samples as well [15, 30]. On the other hand, scales are also widely used to evaluate individual life histories and living conditions of fish by determining their growth dynamics [25] and identifying diseases [19].

Some researchers argue that most of intraspecific variations in shapes of scales and other hard morphological structures could simply be explained by phenotypic plasticity [16], and actually, the relative importance of genetic and environmental factors on scale-morphology is still not exactly known. Some studies have already addressed the questions whether the differences observed in the scale shape could be attributed to differences in life histories of populations, and whether environmental factors, such as recovering food quantity (i.e. compensatory growth) [12], or cadmium treatment $[26,34]$ could affect the reliability of scale shape based stock identifications [12]. However, because of the complex effect of numerous factors and the high degree of genetic diversity, it is generally difficult to evaluate the relative importance of specific factors based on field samples [12]. Nevertheless, no controlled laboratory experiment has yet been reported on the potential role of environmental factors in formation of scale shape. According to the results on other morphological features [9, $20,21]$, it is very likely, however, that scale shape might also vary along environmental gradients.

In this study laboratory experiments were carried out to investigate whether environmental factors, namely the food supply, could affect scale shape during the ontogeny in fish. Two genetically separated, inbred zebrafish, Danio rerio (Hamilton 1822) stocks (Fig. 1) and two feeding protocols were compared in order to assess the role of genetic and environmental components in scale shape variability. Zebrafish is especially a suitable model organism for controlled laboratory investigations, as it has well-known environmental needs [14], reaches the adult size rapidly, after 12 weeks, and the optimal dietary needs are known for the whole life cycle [14].

Specific hypotheses of this study were that 1 . the genetic background has a detectable influence on the scale shape; 2 . the feeding conditions during the ontogeny affect the scale shape with greater impact; and 3. with the improvement of food supply the scale shape could be recovered. 


\section{MATERIALS AND METHODS}

\section{Experimental stocks and design}

Zebrafish were maintained in a recirculating system (Tecniplast) (temperature $=$ $=25 \pm 0.5^{\circ} \mathrm{C}, \mathrm{pH}=7.4 \pm 0.2$, conductivity $=525 \pm 50 \mu \mathrm{S}$; mean $\pm \mathrm{SD}$ ) under a light cycle of 14 hours light and 10 hours dark, and in 30 individuals per 3.5 liters density.

Table 1

Feeding protocols applied in the experiments

\begin{tabular}{|l|l|l|}
\hline \multicolumn{1}{|c|}{ Age of fish } & \multicolumn{1}{|c|}{ Control regimen } & \multicolumn{1}{c|}{ Reduced regimen } \\
\hline 1st and 2nd weeks & $\begin{array}{l}\text { twice a day SDS } 100 \text { and freshly hatched } \\
\text { Artemia nauplii }\end{array}$ & once in every second day SDS 100 \\
\hline 3rd to 5th weeks & $\begin{array}{l}\text { twice a day SDS } 200 \text { and freshly hatched } \\
\text { Artemia nauplii }\end{array}$ & once in every second day SDS 200 \\
\hline 6th to 7th weeks & $\begin{array}{l}\text { twice a day SDS } 300 \text { and freshly hatched } \\
\text { Artemia nauplii }\end{array}$ & once in every second day SDS 300 \\
\hline 8th to 12th weeks & $\begin{array}{l}\text { twice a day SDS 400 and freshly hatched } \\
\text { Artemia nauplii }\end{array}$ & once in every second day SDS 400 \\
\hline After 12th weeks & $\begin{array}{l}\text { twice a day SDS Small Gran and freshly } \\
\text { hatched Artemia nauplii }\end{array}$ & $\begin{array}{l}\text { once in every second day SDS } \\
\text { Small Gran }\end{array}$ \\
\hline
\end{tabular}

Fish were fed with SDS (Special Diets Services Limited International Dietex GB) dry food of increasing granulate size (SDS 100-400 and SDS Small Gran) supplemented with live Artemia nauplii (SERA $\mathrm{GmbH})$. The remaining food was removed one hour after each feeding. Time is calculated from the fertilization.

Table 2

Experimental design

\begin{tabular}{|l|c|c|c|c|}
\hline \multicolumn{1}{|c|}{ Group name } & Stock & Feeding regimen & Sample size & Rearing time \\
\hline AB & AB line & Control & 99 & 12 weeks \\
\hline LF BASKA & LF BASKA stock & Control & 99 & 12 weeks \\
\hline N1 & AB line & Control & 20 & 12 weeks \\
\hline H1 & AB line & Reduced & 20 & 12 weeks \\
\hline N2 & AB line & Control & 20 & 12 weeks \\
\hline H2 & AB line & Reduced & 20 & 12 weeks \\
\hline N3 & AB line & Control & 20 & 12 weeks \\
\hline H3 & AB line & Reduced & 20 & 12 weeks \\
\hline N4 & AB line & Control & 20 & 12 weeks \\
\hline H4 & AB line & Reduced & 20 & 12 weeks \\
\hline REH2 (originated from H2) & AB line & Control & 20 & 12 weeks \\
\hline REH3 (originated from H3) & Ab line & Control & 20 & 12 weeks \\
\hline
\end{tabular}

Description of feeding protocols is given in Table 1. 


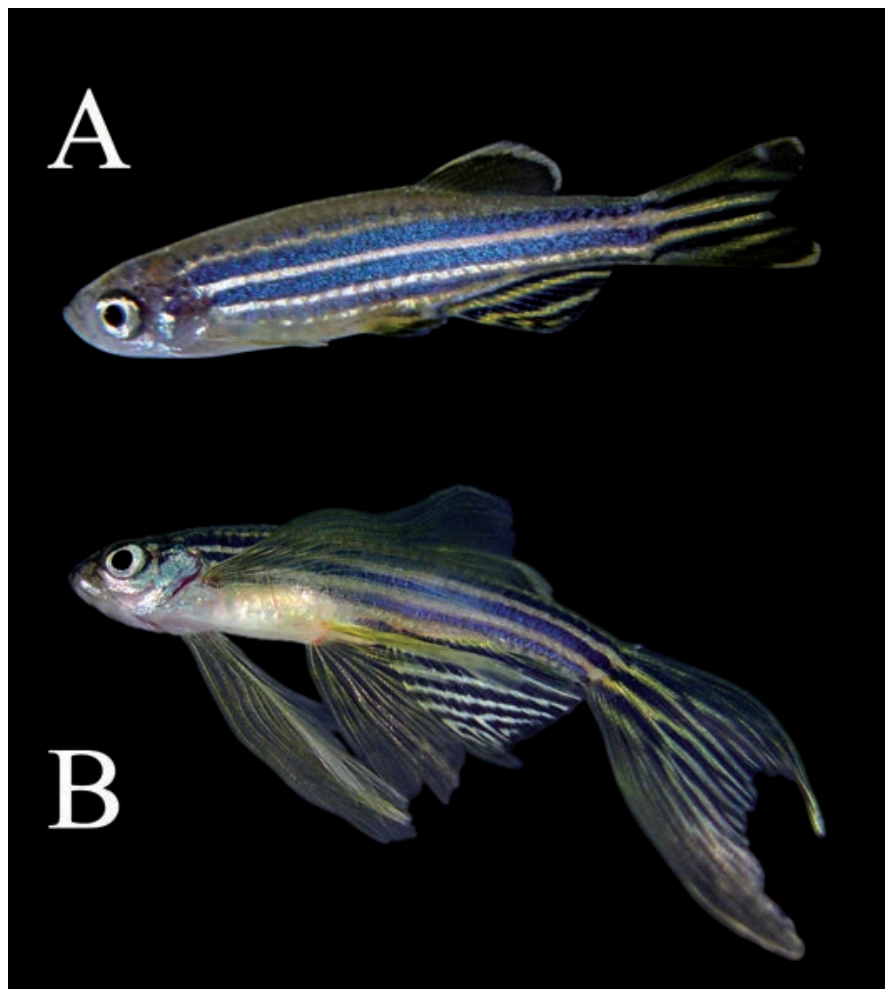

Fig. 1. Investigated zebrafish stocks: A) AB line; B) LF BASKA

To determine the genetic impact on scale shape, zebrafish specimens from a homogeneous registered line (AB line) and a commercial stock (LF BASKA stock) were compared (Fig. 1). Individuals were kept under the same controlled laboratory conditions and fed according to the control regimen (Table 1).

Two groups were created from the offspring of each of four AB line females (altogether eight experimental groups) originated from a single propagation to examine the environmental effect. Thus, genetic differences between these parallel groups were minimal. Groups labeled with "N" were fed following the control regimen (Table 2) according to their age while groups labeled with " $\mathrm{H}$ " were fed following the reduced regimen (Table 2). Fish were reared for 12 weeks, when they normally became adults. Two $\mathrm{H}$ groups (H2, H3) were kept for another 12 weeks and fed according to the control regimen (Table 2) (REH2, REH3) to examine whether any effects of juvenile starving on scale shape can be compensated later. Description of groups is shown in Table 2. 


\section{Sampling}

Scale samples were collected from 20 individuals of each experimental group. One scale was removed from each specimen, from the flank anterior to the dorsal fin (Fig. 2A) [8].

Scales were then placed between two glass slides and scanned with an HP ScanJet 5300C XPA scanner at 2400 dpi. Seven easily definable landmarks were recorded for each scale using tpsUtil [28] and tpsDig2 [29] softwares (Fig. 2B). Landmarks 1 and 2 are the ventro- and dorso-lateral tips of the anterior portion of the scale, landmarks 3 and 4 are at the boundary between the area covered by the other scales and the exposed area, landmark 5 is positioned at the tip of the posterior portion of the scale, landmark 6 is in the center of the anterior edge of the scale, and landmark 7 is the focus of the scale.

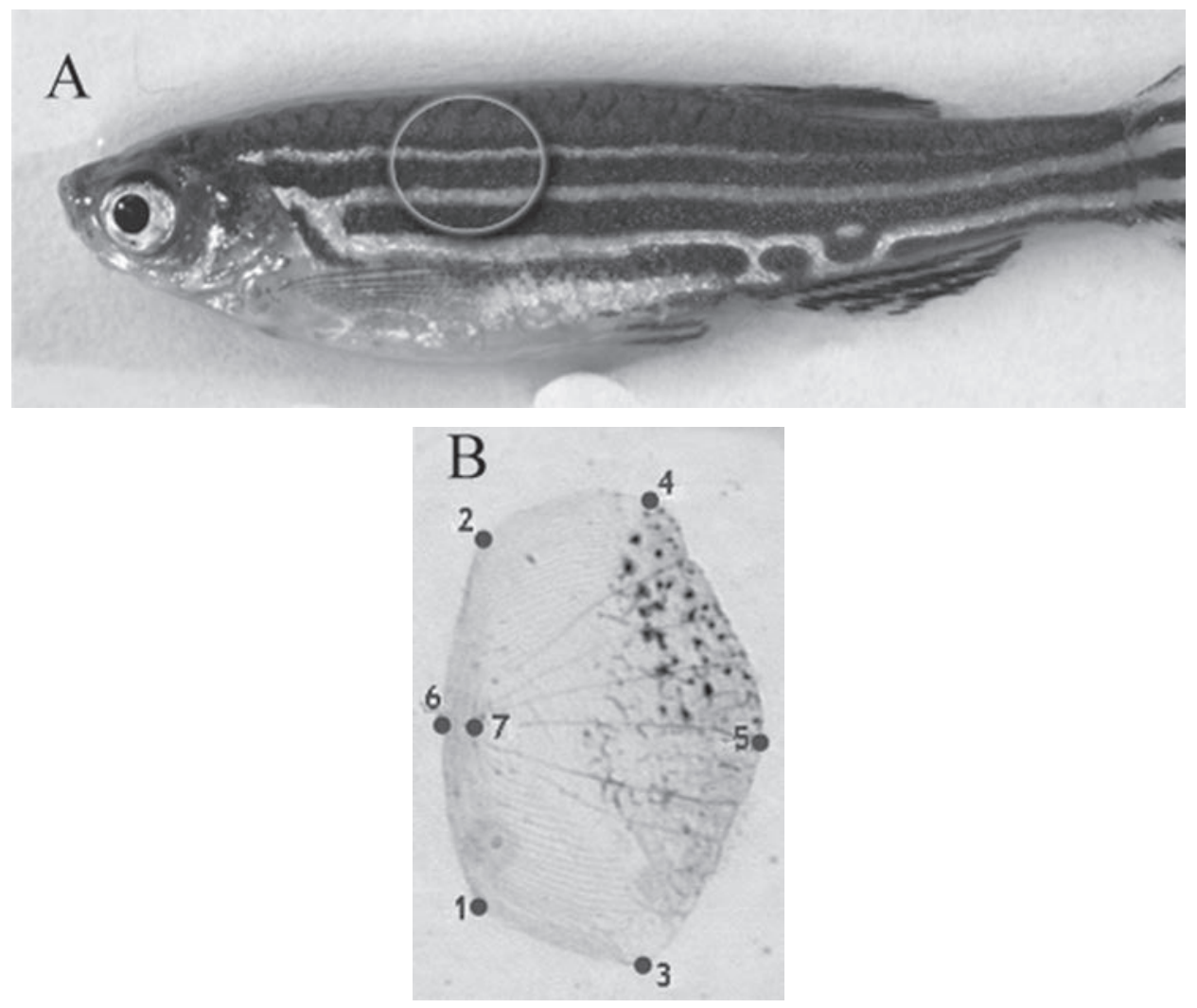

Fig. 2. A) Scale sampling area on zebrafish and B) the recorded scale landmarks 


\section{Statistical analysis}

Scale shape data were processed with the MorphoJ software package [13]. Group identities (ID) were assigned to scales. Scale size was characterized with the scale centroid size, which is the square root of the sum of squared distances between the scale centroid and each landmark, and that is considered as a mathematically shapefree size variable [36]. Generalized least-squares Procrustes superimposition (GLS) was performed on the raw landmarks data on the basis of the principal axis so the landmarks were scaled, rotated and aligned into new shape variables (partial warps, PW), independent of the scale size [27]. A multivariate regression of shape (dependent variable: Procrustes coordinates) on size (independent variable: logarithm of scale centroid size) was performed for each group. Significance of the relationship (i.e. the presence of an allometric effect) was evaluated by using a permutation test against the null hypothesis of independence (10,000 iterations). As data being free of the allometric effects associated with growth, residuals of this regression provided the basis of further analyses [7]. Differentiation of groups was examined with Canonical Variate Analysis (CVA) and Discriminant Function Analysis (DFA). In all cases, a permutation test (10,000 iterations) was performed to test the reliability of results. In case of DFA, cross-validation was also made to test the reliability of classification. For better visibility of the results, averages of the groups were plotted on graphs. Group comparisons from the investigation of the diet impact were classified into five types ("group type"), according to the group relations tested (N vs. N, N vs. H, H vs. $\mathrm{H}, \mathrm{N}$ vs. REH, and REH vs. H). One-way ANOVAs were performed to test the significance of distance data (T-square statistics, Mahalanobis distances) of each group type, and the homogeneity of variances was also tested to determine the appropriate type of post-hoc tests. Since the variances proved to be equal across the compared groups, thus the Tukey HSD test was used for the post-hoc comparisons.

\section{RESULTS}

Regression of scale shape (Procrustes coordinates) on scale centroid size indicated notable allometry (i.e. dependence of shape on size) in all cases. The predicted percentage of the total variation in scale shape accounted for by the allometric effect was $7.7 \%(p<0.001)$ in the experiment comparing AB line versus LF Baska stock and $24.3 \%(p<0.001)$ in the experiment comparing N, H and REH treatment groups. Therefore, controlling scale shape data for the scale size effect was necessary in all further analyses.

\section{Differences between stocks}

The two zebrafish stocks, the AB line and the LF BASKA stock, kept under the same, optimal conditions, could be distinguished with medium reliability based on scale 
Table 3

Classification rates and significance of the discrimination of the experimental zebrafish groups

\begin{tabular}{|l|c|c|c|c|c|}
\hline \multirow{2}{*}{ Groups compared } & \multirow{2}{*}{$\begin{array}{c}\text { Sample } \\
\text { size per } \\
\end{array}$} & \multicolumn{2}{|c|}{ Pure classification } & \multicolumn{2}{c|}{ Cross-validated classification } \\
\cline { 3 - 6 } & group & Rate $(\%)$ & $\chi^{2}(\mathrm{p})$ & Rate $(\%)$ & $\chi^{2}(\mathrm{p})$ \\
\hline AB vs. LF BASKA & 99 & 81.3 & $77.7(<0.001)$ & 78.8 & $65.7(<0.001)$ \\
\hline N1 vs. H1 & 20 & 90.0 & $25.6(<0.001)$ & 72.5 & $8.3(0.004)$ \\
\hline N2 vs. H2 & 20 & 100.0 & $40.0(<0.001)$ & 95 & $32.7(<0.001)$ \\
\hline N3 vs. H3 & 20 & 100.0 & $40.0(<0.001)$ & 97.5 & $36.2(<0.001)$ \\
\hline N4 vs. H4 & 20 & 100.0 & $40.0(<0.001)$ & 100.0 & $40.0(<0.001)$ \\
\hline N2 vs. REH2 & 20 & 90.0 & $25.6(<0.001)$ & 75.0 & $19.6(<0.001)$ \\
\hline H2 vs. REH2 & 20 & 100.0 & $40.0(<0.001)$ & 95.0 & $32.7(<0.001)$ \\
\hline N3 vs. REH3 & 20 & 95.5 & $33.2(<0.001)$ & 93.0 & $28.9(<0.001)$ \\
\hline H3 vs. REH3 & 20 & 100.0 & $40.0(<0.001)$ & 95.5 & $33.2(<0.001)$ \\
\hline
\end{tabular}

Explanations for group names are given in Table 2.

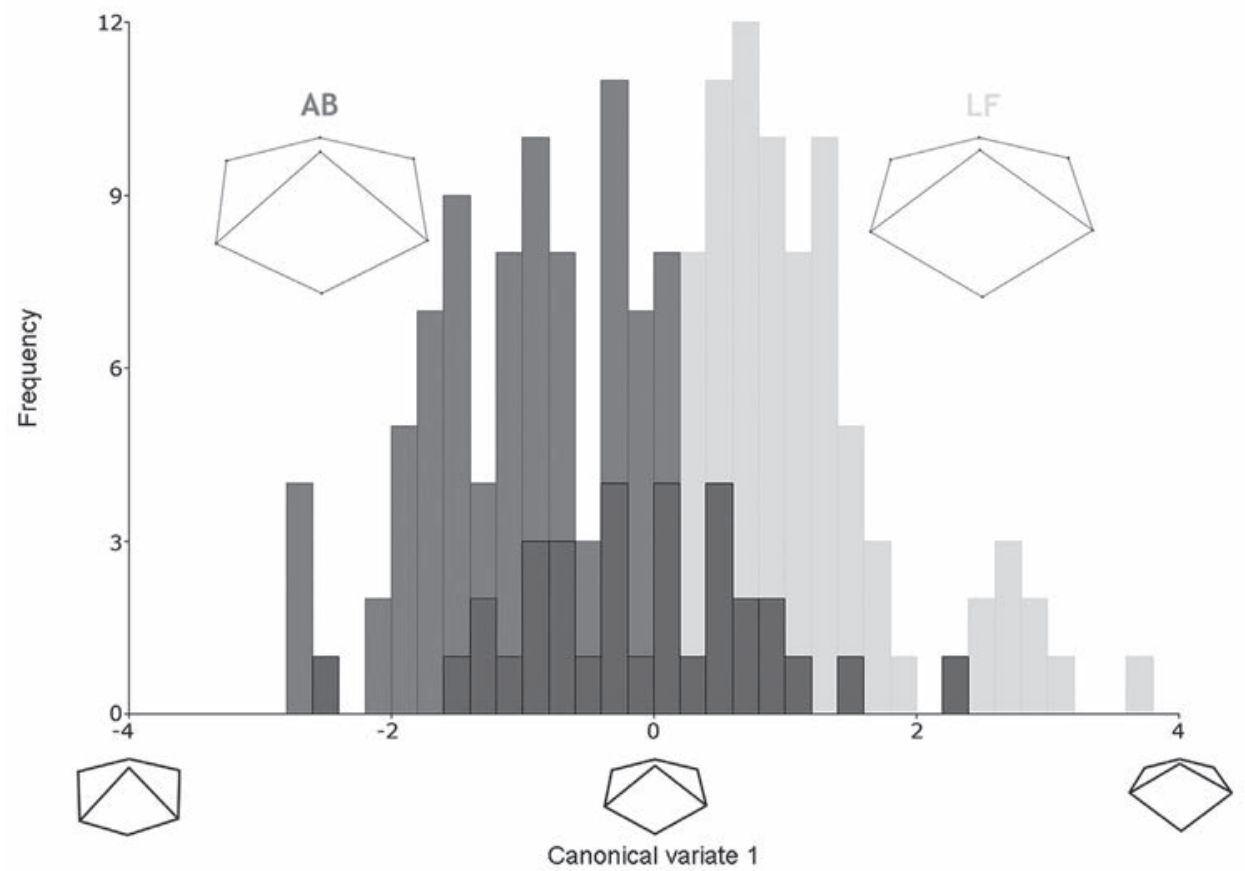

Fig. 3. Average scale shape differences between the AB (grey columns) and LF BASKA (light grey columns) zebrafish stocks according to the Canonical Variate Analysis. The dark columns indicate overlaps between the two groups 


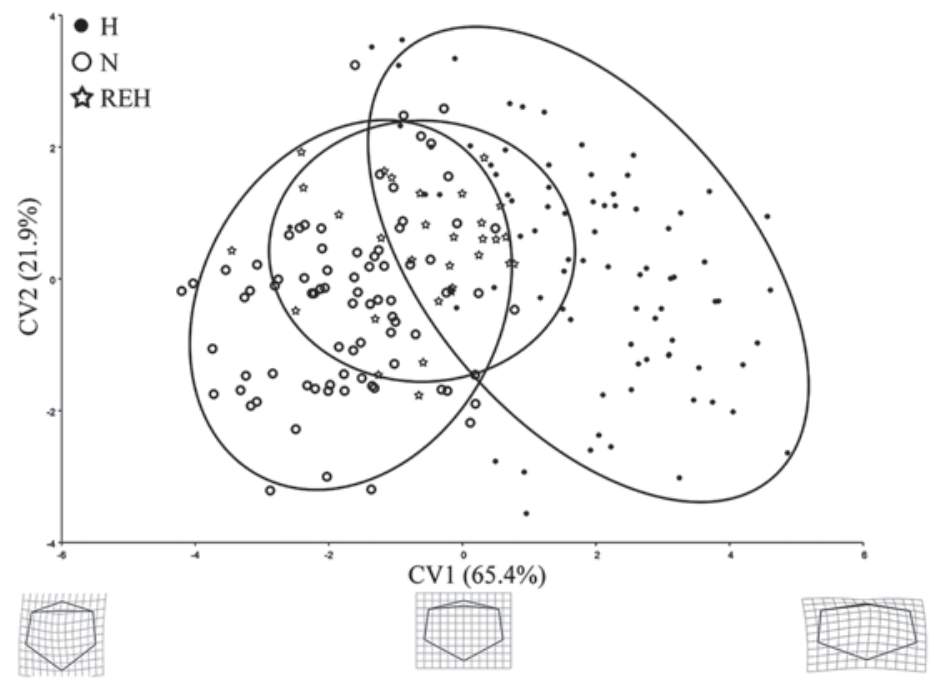

Fig. 4. Canonical Variate Analysis plot comparing scale shapes of zebrafish kept on optimal (N) and reduced $(\mathrm{H})$ diets, and on reduced diet followed by optimal diet $(\mathrm{REH})$

shape. The average shape and the separation of the groups are shown in Figure 3. The main differences between the two groups were in landmarks 3 and 4, which means that the exposed area was bigger in the LF BASKA stock and bigger area covered by other scales in $\mathrm{AB}$ line.

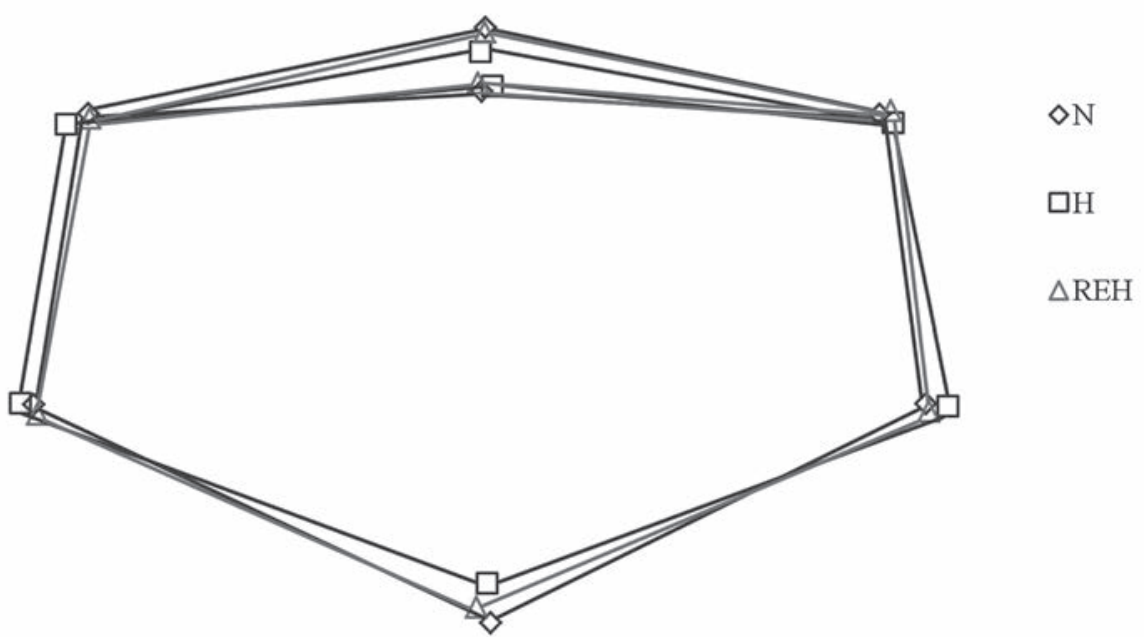

Fig. 5. The mean scale shapes of zebrafish reared on optimal $(\mathrm{N})$, reduced $(\mathrm{H})$ and reduced diet followed by optimal diet (REH) 

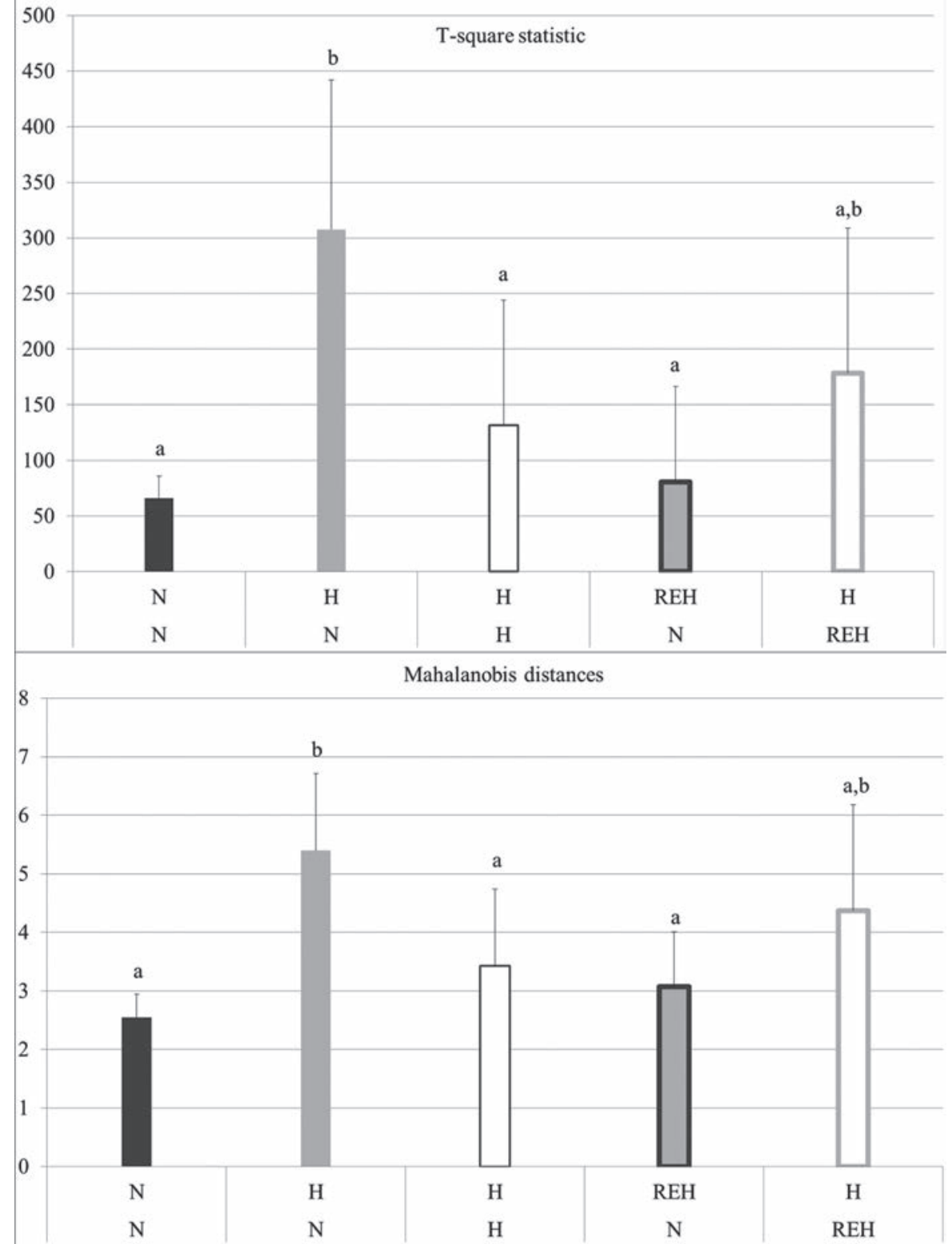

Fig. 6. Differences among treatment groups based on the scale shape of zebrafish according to T-square statistics $(\mathrm{T})$ and Mahalanobis distances (D) (mean $\pm \mathrm{SD}$ ). Values marked with different letters are statistically different at $p<0.05$ according to the one-way ANOVA (for T-square statistic: $\mathrm{F}_{4,39}=9.2, p<0.001$; for Mahalanobis distances: $\mathrm{F}_{4,39}=8.4, p<0.001$ ) followed by Tukey HDS post-hoc test. $\mathrm{N}$ - optimal diet; $\mathrm{H}$ - reduced diet; $\mathrm{REH}$ - reduced diet followed by optimal diet 
Mahalanobis distance (D) between the two groups was 1.5 and indicated a high reliability based on the permutation test $(p<0.001)$. The T-square $(115.2)$ statistics showing average distances of groups from the full sample also showed high reliability $(p<0.001)$. According to the validation results of the DFA, scale shape based group classification showed $81 \%$ identity with real groups on average (cross-validated rate was $78.8 \%$ ) (Table 3 ).

\section{Differences between feeding regimes}

Treatment groups reared on different diets could successfully be distinguished based on scale shape. The CVA-plot (Fig. 4) shows that the $\mathrm{H}$ and $\mathrm{N}$ groups separated well from each other, while the REH group positioned between the two former groups.

Between groups Mahalanobis distances were: $3.9 \pm 1.4$ (mean \pm SD) for $\mathrm{N}_{\mathrm{x}} \mathrm{vs} . \mathrm{H}_{\mathrm{x}}$, $2.8 \pm 1.9$ for $\mathrm{N}_{\mathrm{x}}$ vs. $\mathrm{REH}_{\mathrm{x}}$ and $3.2 \pm 0.6$ for $\mathrm{H}_{\mathrm{x}}$ vs. $\mathrm{REH}_{\mathrm{x}}$ comparisons, respectively. The mean T-square statistic values between the groups were $266.1 \pm 141.5$ for $\mathrm{N}_{\mathrm{x}} \mathrm{vs}$. $\mathrm{H}_{\mathrm{x}}, 101.2 \pm 59.8$ for $\mathrm{N}_{\mathrm{x}}$ vs. $\mathrm{REH}_{\mathrm{x}}$ and $168.0 \pm 55.3$ for $\mathrm{N}_{\mathrm{x}}-\mathrm{REH}_{\mathrm{x}}$ comparisons, respectively.

Validation results (Table 3) show that the N, H and REH groups could successfully be classified with an average rate of $96.9 \%$ (cross-validated rate was $90.4 \%$ ). The mean scale shapes of groups are shown in Figure 5 . The main differences between groups were that $\mathrm{H}$ fish had landmarks 6 and 5 closer to each other reflecting a cranio-caudaly flattened scale shape compared to $\mathrm{N}$ fish. Scale shape of REH fish proved to be intermediate between scales shapes of $\mathrm{N}$ and $\mathrm{H}$ group members.

Mahalanobis distance test results for between group types comparisons are shown in Figure 6. Distances between $\mathrm{N}$ and $\mathrm{H}$ groups were significantly greater than the distances within the $\mathrm{N}$-groups, $\mathrm{H}$-groups and between the $\mathrm{N}$ and REH-groups, either by using T-square statistics $\left(\mathrm{F}_{4,39}=9.2, p<0.001\right)$ or Mahalanobis distances $\left(\mathrm{F}_{4,39}=8.4, p<0.001\right)$. However, none of the distances representing the above relations differed significantly from the distances characterizing the $\mathrm{H}$ vs. REH groups relations.

\section{DISCUSSION}

Based on positive field experiences, scale shape analysis has recently become a widely used tool for differentiating among populations or stocks of fish species [8, 10-12, 22-24], for all that the background of these differences is still not exactly understood. In this study, it was shown, however, that both genetic and environmental factors contribute to intraspecific variability in scale shape of fish and might induce comparable differences.

Our first experiment proved that genetically different zebrafish stocks may be separated based on the shape of their scales. This result supports that intraspecific variability of scale shape of fish has a strong genetic component and genetically iso- 
lated populations of fish might have different scale shape patterns in the wild as well. Genetic divergence among metapopulations of fish could successfully be captured earlier in body shape. For example, Marcil et al. [16] documented that genetic divergence between spawning aggregations of Atlantic cod, Gadus morhua L. 1758 caused detectable morphological differences even at small spatial scales $(<100 \mathrm{~km})$.

Our second experiment proved that the food supply, which is one of the most important environmental factors effecting natural fish populations, can also notably influence the shape of the scale of fish. In zebrafish, scales get flattened in the craniocaudal direction which cannot be fully recovered after the normalization of feeding conditions. A strong environmental influence seems to be common in morphological characters of fish. Amongst the potential environmental components that affect morphological phenotype, the roles of temperature [16] and feeding conditions $[4,16]$ are best documented. The composition and the amount of food consumed evidently influence the conditional state, and especially the extent of the fat reserve of fish, which in turn affects the body shape [4]. Condition of fish (fish mass relative to fish length) is, however, may change dynamically during the life-span and not only due to the variations in the food resource but also by individual feeding strategies, diseases, ontogenetic stages, and even seasonally according to the reproductive and wintering cycle. Several studies have investigated the effects of starvation on body shape [4, 6, 20, 21, 32, 34]. These studies shown consistent changes in body parameters related to the condition and fat metabolism of the examined individual, like body depth, and the largest fat depots in the caudal and trunk region [4]. Body shape parameters that are influenced by the conditional state of fish might therefore limitedly be applicable for intraspecific stock discriminations. Moreover, according to the above reasons, body level morphometric analyses can also be used in a limited way to assess the general environmental characteristic of the habitat from the sample originated.

Compared to the shape of the whole body, scale shape is presumably less sensitive to short-term environmental effects and instantaneous processes, as well as it is less dependent upon the conditional state of fish. In accordance with the observations of Ibáñez et al. [12], present results showed that although scale shape might also recover partly during the compensatory growth (i.e. with the normalization of feeding conditions), this process is much slower and presumably is not as complete as it is in condition related body shape parameters. Moreover, the ring structure of scales conserves individual life histories of fish, and therefore, by a detailed analysis of scale shape by annuli might provide an excellent possibility of investigating variability of environmental impacts and individual life histories both within and among stocks of fish.

Experiments with the zebrafish proved that intraspecific scale shape variations are generated by the interactions of genetic and environmental factors and reflect phenotypic plasticity. Accordingly, information that can be obtained from the morphological analysis of scale samples collected in the field are generally inappropriate to clarify whether the deviation found between scale shapes of two stock of the same species could came from genetic or environmental differences [see also 18]. 
Although, in intraspecific studies, shape analysis of scales seems to have the same limits as the shape analysis of the whole body, namely based on these analyses only, no decision can be made on the relative importance of genetic and environmental factors being responsible for differences among groups, the former method still bears several advantages. Scale sampling is not as stressful for fish as whole body investigation, and therefore, the introduction of the method is highly recommended when investigating protected or endangered fish species. In addition, as the scale method is much easier, time and cost efficient, than the traditional whole body methods, it may be favorable in other cases as well. However, the scale method is not applicable for all fish species. Species that do not have scales (e.g. acipenseroids) or have very small scales [e.g. European eel, Anguilla anguilla (L. 1758)] can only be examined by the traditional, full-body inspection or examination of other hard formulas (e.g. otolith [1]), where the individual does not survive the investigation.

To conclude, genetically and dietetically different experimental groups of zebrafish could successfully be distinguished according to the shape of their scales, and the results showed that both genetic and environmental factors may notably influence scale shape formation. It is suggested that scale shape analysis might be used as an explanatory tool to detect potential intraspecific variability of environmental influences impacting genetically homogeneous groups of individuals. However, results also indicated that due to its sensitivity to environmental factors, the applicability of a morphometric scale analysis in identifying intraspecific stock membership could be limited. In order to improve the applicability of the method and to assess its potentials, more laboratory inventories are needed testing the type and extent of effects that the most important environmental stressors (e.g. food, temperature, $\mathrm{pH}$ ) might have on scale shape.

\section{ACKNOWLEDGEMENTS}

This work was financially supported by the research funding programme "KMOP-1.1.1-09/1-2009-0048" National Office for Research and Technology of Hungary, "Sustainable conservation on Hungarian Natura 2000 Sites-SH/4/8" Swiss Contribution to Hungary, "KMR-12-1-2012-0221" and Bolyai János research grant by the Hungarian Academy of Sciences - "BO/00054/12/4".

\section{REFERENCES}

1. Agüera, A., Brophy, D. (2011) Use of saggital otolith shape analysis to discriminate Northeast Atlantic and Western Mediterranean stocks of Atlantic saury, Scomberesox saurus saurus (Walbaum). Fish Res. 110, 465-471.

2. Beacham, T. D. (1990) A genetic analysis of meristic and morphometric variation in chum salmon (Oncorhynchus keta) at three different temperatures. Can. J. Zool. 68, 225-229.

3. Brönmark, C., Miner, J. G. (1992) Predator-induced phenotypical change in body morphology in Crucian carp. Science 258, 1348-1350.

4. Currens, K. P., Sharpe, C. S., Hjort, R., Schreck, C. B., Li, H. W. (1989) Effects of different feeding regimes on the morphometrics of chinook salmon (Oncorhynchus tshawytscha) and rainbow trout (O. mykiss). Copeia 3, 689-695. 
5. Day, T., Pritchard, J., Schluter, D. (1994) A comparison of two sticklebacks. Evolution 48, 1723-1734.

6. Ehrlich, K. F., Blaxter, J. H. S., Pemberton, R. (1976) Morphological and histological changes during the growth and starvation of herring and plaice larvae. Mar. Biol. 35, 105-118.

7. Elmer, K. R., Kusche, H., Lehtonen, T. K., Meyer, A. (2010) Local variation and parallel evolution: morphological and genetic diversity across a species complex of neotropical crater lake cichlid fishes. Philos. Trans. R. Soc. Lond. B. Biol. Sci. 365, 1763-1782.

8. Garduño-Paz, M. V., Demetriou, M., Adams, C. E. (2010) Variation in scale shape among alternative sympatric phenotypes of Arctic charr Salvelinus alpinus from two lakes in Scotland. J. Fish Biol. 76, 1491-1497.

9. Georgakopoulou, E., Sfakianakis, D. G., Kouttouki, S., Divanach, P., Kentouri, M., Koumoundouros, G. (2007) The influence of temperature during early life on phenotypic expression at later ontogenetic stages in sea bass. J. Fish Biol. 70, 278-291.

10. Ibáñez, A. L., Cowx, I. G., O’Higgins, P. (2007) Geometric morphometric analysis of fish scales for identifying genera, species, and local populations within Mugilidae. Can. J. Fish Aquat. Sci. 64, 1091-1100.

11. Ibáñez, A. L., Cowx, I. G., O’Higgins, P. (2009) Variation in elasmoid fish scale patterns is informative with regard to taxon and swimming mode. Zool. J. Linn. Soc. 155, 834-844.

12. Ibáñez, A. L., Pacheco-Almanzar, E., Cowx, I. G. (2012) Does compensatory growth modify fish scale shape? Environ. Biol. Fishes 94, 477-482.

13. Klingenberg, C. P. (2011) MorphoJ: an integrated software package for geometric morphometrics. Mol. Ecol. Resour. 11, 353-357.

14. Lawrence, C. (2007) The husbandry of zebrafish (Danio rerio): A review. Aquaculture 269, 1-20.

15. Leak, A. (1990) Cleaning archaeological fish scales. Circaea 6, 119-120.

16. Marcil, J., Swain, D. P., Hutchings, J. A. (2006) Genetic and environmental components of phenotypic variation in body shape among populations of Atlantic cod (Gadus morhua L.). Biol. J. Linn. Soc. Lond. 88, 351-365.

17. Mérigot, B., Letourneur, Y., Lecomte-Finiger, R. (2007) Characterization of local populations of the common sole Solea solea (Pisces, Soleidae) in the NW Mediterranean through otolith morphometrics and shape analysis. Mar. Biol. 151, 997-1008.

18. Mittelbach, G. G., Osenberg, C. W., Wainwright, P. C. (1999) Variation in feeding morphology between pumpkinseed populations: Phenotypic plasticity or evolution? Evol. Ecol. Res. 1, 111-128.

19. Noga, E. J. (2010) Fish Disease - Diagnosis and Treatment Second Edition. Wiley-Blackwell, USA.

20. Park, I.-S., Im, J. H., Ryu, D. K., Nam, Y. K., Kim, D. S. (2001) Effect of starvation on morphometric changes in Rhynchocypris oxycephalus (Sauvage and Dabry). J. Appl. Ichthyol. 17, 277-281.

21. Park, I.-S., Woo, S. R., Song, Y.-C., Cho, S. H. (2007) Effects of starvation on morphometric characteristics of olive flounder, Paralichthys olivaceus. Ichthyol. Res. 54, 297-302.

22. Poulet, N., Reyjol, Y., Collier, H., Lek, S. (2005) Does fish scale morphology allow the identification of populations at a local scale? A case study for rostrum dace Leuciscus leuciscus burdigalensis in River Viaur (SW France). Aquat. Sci. 67, 122-127.

23. Richards, R. A., Esteves, C. (1997) Stock-specific variation in scale morphology of Atlantic striped bass. Trans. Am. Fish Soc. 126, 908-918.

24. Richards, R. A., Esteves, C. (1997) Use of scale morphology for discriminating wild stocks of Atlantic striped bass. Trans. Am. Fish Soc. 126, 919-925.

25. Ricker, W. E. (1975) Computation and interpretation of biological statistics of fish populations. Bull. Fish Res. Board. Can. 191, 1-382.

26. Rishi, K. K., Jain, M. (1998) Effect of toxicity of cadmium on scale morphology in Cyprinus carpio (Cyprinidae). Bull. Environ. Contam. Toxicol. 60, 323-328.

27. Rohlf, F. J. (1990) Morphometrics. Annu. Rev. Ecol. Syst. 21, 299-316.

28. Rohlf, F. J. (2010) tpsUtil, file utility program. version 1.46. Department of Ecology and Evolution, State University of New York at Stony Brook. 
29. Rohlf, F. J. (2010) tpsDig2, digitize landmarks and outlines, version 2.16. Department of Ecology and Evolution, State University of New York at Stony Brook.

30. Slyke, N. V. (1998) A review of the analysis of fish remains in Chumash sites. Pac. Coast. Archaeol. Soc. Q. 34, 25-58.

31. Šumer, S., Kováč, V., Povž, M., Slatner, M. (2005) External morphology of a Slovenian population of pumpkinseed Lepomis gibbosus (L.) from a habitat with extreme thermal conditions. J. Appl. Ichthyol. 21, 306-311.

32. Theilacker, G. H. (1978) Effect of starvation on the histological and morphological characteristics of jack mackerel, Trachurus symmetricus, larvae. Fish Bull. 76, 403-414.

33. Verhaegen, Y., Adriaens, D., De Wolf, T., Dhert, P., Sorgeloos, P. (2007) Deformities in larval gilthead sea bream (Sparus aurata): A qualitative and quantitative analysis using geometric morphometrics. Aquaculture 268, 156-168.

34. Yin, M. C., Blaxter, J. H. S. (1986) Morphological changes during growth and starvation of larval cod (Gadus morhua L.) and flounder (Platichthys flesus L.). J. Exp. Mar. Bio. Ecol. 104, 215-228.

35. Yoshitomi, T., Koyama, J., Iida, A., Okamoto, N., Ikeda, Y. (1998) Cadmium-induced scale deformation in carp (Cyprinus carpio). Bull. Environ. Contam. Toxicol. 60, 639-644.

36. Zelditch, M. L., Swiderski, D. L., Sheets, H. D., Fink, W. L. (2004) Geometric Morphometrics for Biologists: A Primer. Elsevier/Academic Press, Amsterdam. 